version of 2 April 2015

\title{
Vacuum energy density and pressure of a massive scalar field
}

\author{
Fernando Daniel Mera ${ }^{1}$ and S A Fulling ${ }^{2}$ \\ ${ }^{1}$ Department of Physics, Northeastern University, Boston, MA 02115 USA \\ 2 Departments of Mathematics and Physics, Texas A\&M University, College Station, \\ TX 77843-3368 USA
}

\begin{abstract}
With a view toward application of the Pauli-Villars regularization method to the Casimir energy of boundaries, we calculate the expectation values of the components of the stress tensor of a confined massive field in $1+1$ space-time dimensions. Previous papers by Hays and Fulling are bridged and generalized. The Green function for the time-independent Schrödinger equation is constructed from the Green function for the whole line by the method of images; equivalently, the onedimensional system is solved exactly in terms of closed classical paths and periodic orbits. Terms in the energy density and in the eigenvalue density attributable to the two boundaries individually and those attributable to the confinement of the field to a finite interval are distinguished so that their physical origins are clear. Then the pressure is found similarly from the cylinder kernel, the Green function associated most directly with an exponential frequency cutoff of the Fourier mode expansion. Finally, we discuss how the theory could be rendered finite by the Pauli-Villars method.
\end{abstract}

PACS numbers: 03.70.+k, 11.10.Gh

AMS classification scheme numbers: $81 \mathrm{~T} 55$ 


\section{Introduction}

The Casimir energy [1, 2, 3] of a massive scalar field in two space-time dimensions, despite the seeming simplicity of the model, has not been completely studied. The 1979 paper of Hays [4] calculated the energy and the force but did not look at the local energy density, a subject of much interest today. The more recent paper of Fulling [5] treated the energy density for a massless scalar field from a viewpoint of spectral theory and asymptotics, but did not consider the massive field. Neither paper calculated pressure directly. The present article generalizes the works [4] and [5] and uses methods from each.

The primary reason for studying massive fields in this context is to be able to conduct a Pauli-Villars regularization [6, 7, 8, 9, 10, (see Appendix A). It has become clear [11] that the traditional ultraviolet cutoff produces unphysical results, dependent on the direction of "point-splitting", for the counterterms in energy density and pressure near perfectly reflecting boundaries; this development casts some doubt on the claim that such approaches to divergences are more "physical" than the analytic ones (dimensional or zeta). The Pauli-Villars method (which occupies a place somewhere between the analytic and the cutoff methods) preserves Lorentz invariance, and hence one hopes that it will avoid this problem. A serious implementation of this strategy requires calculations in four space-time dimensions, which are deferred to future work, but here we give it a test drive. The previous applications of the method that are most pertinent to our problem are those to gravitational backgrounds, and we review the relevant literature in Appendix A.

In section 2 the local energy densities $E_{\text {Weyl }}(t), E_{\text {per }}(t)$, and $E_{\text {bdry }}(x, t)$, related respectively to zero-length, periodic, and closed reflected classical paths, are expressed in terms of Macdonald functions. (Here $t$ is a temporary regularization parameter.) These are expanded in various limits in section 3. As expected, the $m \rightarrow 0$ limit reproduces the known theory of the massless field; the $t \rightarrow 0$ and $m \rightarrow \infty$ limits provide needed input into the Pauli-Villars construction. Section 4 deals with the (regularized) total energy and its nontrivial relation to the nonconstant density term, $E_{\mathrm{bdry}}(x, t)$. Section 5 deals with the eigenvalue density and counting function. Section 6 and section 8 use the cylinder-kernel method pioneered by Hays [12, 4] to find the various contributions to the expectation value of the pressure; section 7 presents the dependence on the parameter $\xi$ that labels different possible gravitational couplings. Finally, section 9 applies the Pauli-Villars procedure.

The key results of the paper are the formulas (12), (16), and (17) for energy density; (44), (47), and (49) for pressure; and (51) for the conformal correction to the energy density (the correction to the pressure being zero). 


\section{Vacuum energy density from closed and periodic orbits}

We consider a finite interval with either a Dirichlet or a Neumann boundary condition at each end, following the notation of [5], which allows the two boundary conditions to be treated simultaneously. Thus $H=-\frac{d^{2}}{d x^{2}}+m^{2}$ acts in $L^{2}(0, L)$ on the domain defined by

$$
u^{(1-l)}(0)=0, \quad u^{(1-r)}(L)=0, \quad l, r \in\{0,1\} .
$$

The superscript is the number of derivatives in the boundary condition. Thus $l=0$ means that the left endpoint is Neumann, etc. In nonrelativistic terms we are solving a Schrödinger equation with potential $V=m^{2}$. The Green function can be constructed from $G_{\infty}$, the Green function on the whole real line, by the method of images:

$$
\begin{aligned}
G\left(\omega^{2}, x, y\right)= & G_{\infty}(y)+(-1)^{l} G_{\infty}(-y)+(-1)^{r} G_{\infty}(2 L-y)+(-1)^{l+r} G_{\infty}(2 L+y) \\
& +(-1)^{l+r} G_{\infty}(-2 L+y)+(-1)^{2 l+r} G_{\infty}(-2 L-y) \\
& +(-1)^{l+2 r} G_{\infty}(4 L-y)+(-1)^{2 l+2 r} G_{\infty}(4 L+y)+\cdots \\
= & \sum_{n=0}^{\infty}(-1)^{n(l+r)} G_{\infty}(-2 n L+y)+\sum_{n=0}^{\infty}(-1)^{l+n(l+r)} G_{\infty}(-2 n L-y) \\
& +\sum_{n=1}^{\infty}(-1)^{-l+n(l+r)} G_{\infty}(2 n L-y)+\sum_{n=1}^{\infty}(-1)^{n(l+r)} G_{\infty}(2 n L+y) .
\end{aligned}
$$

(Here and occasionally elsewhere we suppress some function arguments to avoid clutter.) The only difference from [5] is that in $G_{\infty}$ the energy parameter $\lambda$ must be replaced by $\lambda-m^{2}$. Thus, many formulas in [5] remain valid if we replace $\omega(\equiv \sqrt{\lambda})$ by

$$
\left.\kappa \equiv \sqrt{\omega^{2}-m^{2}} \quad \text { (hence } \omega d \omega=\kappa d \kappa\right),
$$

and the basic Green function is

$$
G_{\infty}\left(\omega^{2}, x, y\right)=\frac{i}{2 \kappa} e^{i \kappa|x-y|} .
$$

It is easy to check from first principles that this new $G_{\infty}$, and hence $G$, satisfy the right equation,

$$
-\frac{\partial^{2} G}{\partial x^{2}}-\kappa^{2} G=\left(H_{x}-\omega^{2}\right) G\left(\omega^{2}, x, y\right)=\delta(x-y) .
$$

The spectral densities in terms of $\lambda$ for this problem are the same as in [5] except for the shift of the argument variable $\lambda$ by $-m^{2}$. This is exactly to be expected, because we know that adding a constant to the potential in the Schrödinger equation merely adds that constant to all the energies. Note that only values of $\omega \geq m$ need to be considered, because we know that $H$ has no spectrum below $m$. This even comes out automatically in the formalism, because if $\kappa$ is imaginary, then the imaginary part of $G_{\infty}$ is zero and doesn't contribute to the density of states. When we go to the variable $\omega$ the situation is slighly more complicated: $\kappa$ is not just $\omega$ minus a constant, and that is where some interesting new behavior arises. 
The density of eigenvalues is given in terms of the Green function by

$$
\sum_{j}^{\infty} \delta\left(\lambda_{j}-\lambda\right)=\frac{1}{\pi} \int_{0}^{L} d x[\operatorname{Im} G(\lambda+i \epsilon, x, y)]_{x=y} .
$$

It is more convenient to work with the density with respect to $\omega=\sqrt{\lambda}$, which carries an additional factor $2 \omega$. Then

$$
\rho(\omega) d \omega=\frac{2 \omega}{\pi} d \omega \int_{0}^{L} d x \operatorname{Im} G\left(\omega^{2}, x, x\right)=\frac{2 \kappa}{\pi} d \kappa \int_{0}^{L} d x \operatorname{Im} G\left(\omega^{2}, x, x\right) .
$$

We have by definition

$$
\rho(\omega)=\int_{0}^{L} d x \sigma(\omega, x), \quad \sigma(\omega, x)=\frac{2 \omega}{\pi} \operatorname{Im} G\left(\omega^{2}, x, x\right),
$$

and hence

$$
\begin{aligned}
\frac{\pi \kappa}{\omega} \sigma(\omega, x)= & 2 \kappa \operatorname{Im} G\left(\omega^{2}, x, x\right) \\
= & \sum_{n=0}^{\infty}(-1)^{n(l+r)} \cos (2 \kappa n L)+\sum_{n=0}^{\infty}(-1)^{l+n(l+r)} \cos (2 \kappa(n L+x)) \\
& +\sum_{n=1}^{\infty}(-1)^{-l+n(l+r)} \cos (2 \kappa(n L-x))+\sum_{n=1}^{\infty}(-1)^{n(l+r)} \cos (2 \kappa n L) \\
= & 1+2 \sum_{n=1}^{\infty}(-1)^{n(l+r)} \cos (2 \kappa n L)+\sum_{n=-\infty}^{\infty}(-1)^{l+n(l+r)} \cos (2 \kappa(x+n L)) \\
\equiv & \frac{\pi \kappa}{\omega}\left(\sigma_{\text {Weyl }}+\sigma_{\text {per }}+\sigma_{\text {bdry }}\right) .
\end{aligned}
$$

The paths connecting $x$ to an image charge in (2) can be folded back into the original interval as paths connecting $x$ to $y$ after some number of reflections from the endpoints. In (9) these paths connect $x$ to itself. The first term, coming from a path of zero length, provides the bulk spectral density of Weyl's famous theorem. Paths with an even number of reflections are periodic and provide a spatially homogeneous Casimir energy. Terms with an odd number of reflections "bounce" off one of the boundaries and yield energy distributions somewhat concentrated there.

The stress tensor of a scalar field contains a free parameter, $\xi$, reflecting an ambiguity in its coupling to the gravitational field. The relevant formulas are reviewed in section 6 and appendix C. Until section [6 we confine attention to the value $\xi=\frac{1}{4}$, for which the energy expressions are maximally simple. In particular, the contribution of the space derivatives to the energy density is identical to that of the time derivatives, so we can write (following [5])

$$
\begin{aligned}
\left\langle T_{00}(t, x)\right\rangle & \equiv E(t, x)=-\frac{1}{2} \frac{d}{d t} \int_{0}^{\infty} \sigma(\omega, x) e^{-\omega t} d \omega \\
& \equiv E_{\mathrm{Weyl}}(t)+E_{\mathrm{per}}(t)+E_{\mathrm{bdry}}(t, x) .
\end{aligned}
$$

Here $t$ is an ultraviolet cutoff parameter, which can be related by a Wick rotation to a difference of physical time coordinates. 
Vacuum energy of a massive scalar field

From [13, (3.914.1)],

$$
\int_{0}^{\infty} e^{-t \sqrt{m^{2}+\kappa^{2}}} \cos (2 n L \kappa) d \kappa=\frac{m t}{\sqrt{t^{2}+(2 n L)^{2}}} K_{1}\left(m \sqrt{t^{2}+(2 n L)^{2}}\right),
$$

where $K$ is a Macdonald function (see Appendix B). In particular, if $n=0$ (the Weyl term), (11) reduces to $K_{1}(m t)$. Thus, doing the change of variables (3), we get

$$
\begin{aligned}
E_{\mathrm{Weyl}}(t) & =-\frac{1}{2} \frac{d}{d t} \int_{0}^{\infty} \sigma_{\mathrm{Weyl}}(\omega) e^{-\omega t} d \omega \\
& =-\frac{1}{2 \pi} \frac{d}{d t} \int_{0}^{\infty} \frac{\sqrt{\kappa^{2}+m^{2}}}{\kappa} \cdot \frac{\kappa}{\sqrt{\kappa^{2}+m^{2}}} e^{-t \sqrt{\kappa^{2}+m^{2}}} d \kappa \\
& =-\frac{1}{2 \pi} \frac{d}{d t} m K_{1}(m t)=\frac{m^{2}}{2 \pi}\left(\frac{1}{m t} K_{1}(m t)+K_{0}(m t)\right)
\end{aligned}
$$

(see (B.3)). Similarly, the periodic term is

$$
\begin{aligned}
E_{\mathrm{per}}(t) & =-\frac{1}{\pi} \frac{d}{d t} \sum_{n=1}^{\infty}(-1)^{n(l+r)} \int_{0}^{\infty} \sigma_{\mathrm{per}}(\omega) e^{-\omega t} d \omega \\
& =-\frac{1}{\pi} \frac{d}{d t} \sum_{n=1}^{\infty}(-1)^{n(l+r)} \frac{m t}{\sqrt{(2 n L)^{2}+t^{2}}} K_{1}\left(m \sqrt{(2 n L)^{2}+t^{2}}\right) .
\end{aligned}
$$

Finally, the boundary term is

$$
\begin{aligned}
E_{\mathrm{bdry}}(t, x) & =-\frac{(-1)^{l}}{2 \pi} \frac{d}{d t} \sum_{n=-\infty}^{\infty}(-1)^{n(l+r)} \int_{0}^{\infty} \frac{\omega}{\kappa} \cos (2 \kappa(x+n L)) e^{-\omega t} d \omega \\
& =-\frac{(-1)^{l}}{2 \pi} \frac{d}{d t} \sum_{n=-\infty}^{\infty}(-1)^{n(l+r)} \frac{m t}{\sqrt{(2(x+n L))^{2}+t^{2}}} K_{1}\left(m \sqrt{(2(x+n L))^{2}+t^{2}}\right) .
\end{aligned}
$$

\section{Asymptotic behaviors}

\subsection{Small $t$}

To put the energy expressions (12)-(14) into the usual form for renormalization

calculations, we need to expand them in power (Laurent) series in $t$. Using (B.1) one gets

$$
\begin{aligned}
E_{\mathrm{Weyl}}(t) & =-\frac{1}{2 \pi} \frac{d}{d t}\left[\frac{1}{t}+\frac{m^{2} t}{2} \ln \left(\frac{m t}{2}\right)+\frac{m^{2} t}{4}(2 \gamma-1)+O\left((m t)^{2}\right)\right] \\
& =\frac{1}{2 \pi}\left[\frac{1}{t^{2}}-\frac{m^{2}}{2} \ln \left(\frac{m t}{2}\right)-\frac{m^{2}}{4}(2 \gamma+1)\right]+O(t) .
\end{aligned}
$$

When the derivatives in (13) and (14) are calculated, only one term survives in the limit $t \rightarrow 0$. Furthermore, the resulting limits are finite (no negative powers or logarithm of $t):$

$$
E_{\mathrm{per}}(0)=-\frac{1}{\pi} \sum_{n=1}^{\infty}(-1)^{n(l+r)} \frac{m}{2 n L} K_{1}(2 n L m)
$$


Vacuum energy of a massive scalar field

$$
E_{\mathrm{bdry}}(0, x)=-\frac{(-1)^{l}}{2 \pi} \sum_{n=-\infty}^{\infty}(-1)^{n(l+r)} \frac{m}{2|x+n L|} K_{1}(2|x+n L| m) .
$$

In the case (14), this argument assumes $x \neq 0$ and $x \neq L$, and the limit is not uniform in distance from the boundary. Therefore, we shall need to revisit this case when considering the total energy in section 4 .

\subsection{Massless limit}

The same expansion (15) shows that when $m=0$,

$$
E_{\text {Weyl }}(t)=\frac{1}{2 \pi t^{2}} \quad(m=0),
$$

as expected [5]. (The only interesting fact is that (15) includes less trivial terms when $m>0$.) Verifying the massless limits of the infinite sums $E_{\text {per }}(0)$ and $E_{\text {bdry }}(0, x)$ is complicated by the conflict between the $m \rightarrow 0$ and $n \rightarrow \infty$ limits in the individual Macdonald functions. However, (16) when $l+r$ is even is a special case of [14, (2.10)], a complicated formula from which only one term survives when $m=0$ :

$$
E_{\text {per }}(0)=-\frac{\pi}{24 L^{2}} \quad(m=0),
$$

the well known one-dimensional Casimir energy. In exactly the same way, [14, (2.12)] gives $E_{\text {per }}(0)=+\pi / 48 L^{2}$ when $l+r$ is odd (one Dirichlet and one Neumann end).

\subsection{Supermassive limit}

The behavior when $m \rightarrow \infty$ is critical for the Pauli-Villars analysis. Using (B.2) one sees that all the limits are zero: From (12) we have

$$
\lim _{m \rightarrow \infty} E_{\mathrm{Weyl}}(t)=\lim _{m \rightarrow \infty} \frac{m^{2}}{2 \pi} \sqrt{\frac{\pi}{2 m t}} e^{-m t}=0
$$

when $t>0$. Similarly, the terms of (16) and (17) (or even (13) and (14)) for fixed $m$ vanish sufficiently rapidly with $n$ to make the series converge, and for fixed $n$ decrease monotonically to 0 as $m \rightarrow \infty$; therefore, by standard arguments [15, pp. 3 and 8] the sum of the series approaches 0 as $m \rightarrow \infty$. The only exceptions are the terms in (17) with $n=0, x=0$ and with $n=-1, x=L$, where the energy density is singular, as previously noted.

\section{Total energy}

The energy equals the integral of the energy density over $x$ from 0 to $L$, at least formally. Departing somewhat from the notation of [5], we denote a total energy by the letter $\bar{E}$. $E_{\text {Weyl }}$ and $E_{\text {per }}$ are constant in $x$, so their energies are obtained by multiplying by $L$ and there is nothing more to be said. 
When $l+r$ is even, the boundary formula (14) yields

$\bar{E}_{\mathrm{bdry}}(t)=-\frac{(-1)^{l}}{2 \pi} \frac{d}{d t} \sum_{n=-\infty}^{\infty} \int_{0}^{L} \frac{m t}{\sqrt{4(x+n L)^{2}+t^{2}}} K_{1}\left(m \sqrt{4(x+n L)^{2}+t^{2}}\right) d x$.

Making a change of variables $x^{\prime}=x+n L$ in each term, we have

$$
\begin{aligned}
\bar{E}_{\mathrm{bdry}}(t) & =-\frac{(-1)^{l}}{2 \pi} \frac{d}{d t} \sum_{n=-\infty}^{\infty} \int_{L n}^{L(n+1)} \frac{m t}{\sqrt{4 x^{\prime 2}+t^{2}}} K_{1}\left(m \sqrt{4 x^{\prime 2}+t^{2}}\right) d x^{\prime} \\
& =-\frac{(-1)^{l}}{2 \pi} \frac{d}{d t} \int_{-\infty}^{\infty} \frac{m t}{\sqrt{4 x^{\prime 2}+t^{2}}} K_{1}\left(m \sqrt{4 x^{\prime 2}+t^{2}}\right) d x^{\prime} .
\end{aligned}
$$

After another change of variables, $u^{2}=4 x^{\prime 2}+t^{2}$, we get with (B.4)

$$
\begin{aligned}
\bar{E}_{\mathrm{bdry}}(t) & =-\frac{(-1)^{l}}{2 \pi} \frac{d}{d t}(m t) \int_{t}^{\infty} \frac{K_{1}(m u)}{\sqrt{u^{2}-t^{2}}} d u \\
& =-\frac{(-1)^{l}}{4} \frac{d}{d t} e^{-m t}=\frac{(-1)^{l}}{4} m e^{-m t} .
\end{aligned}
$$

One can now take $t$ to 0 , obtaining in the Dirichlet case $(l=1)$

$$
\bar{E}_{\text {bdry }}(0)=-\frac{m}{4},
$$

in agreement with Hays [4] and with the conclusion in [5] that the net boundary energy vanishes in the massless case. It definitely does not agree (for any $m$ ) with an attempt to integrate $E_{\mathrm{bdry}}(0, x)$ over the interval (that is, to take the cutoff away before integrating), which encounters divergences at the endpoints. For later use note also that

$$
\lim _{m \rightarrow \infty} \bar{E}_{\mathrm{bdry}}(t)=0 \quad \text { if (and only if) } t>0 .
$$

When $l+r$ is odd, $\bar{E}_{\mathrm{bdry}}(t)$ vanishes for an elementary reason: The middle member of (22) acquires a factor $(-1)^{n}$, and hence term $n$ cancels term $-(n+1)$.

\section{Counting eigenvalues}

For completeness of the comparison with the massless theory in [5], we look here at the global eigenvalue density, $\rho(\omega)$, and its integral, the counting function $N(\omega)$. In section 2 we started from the local spectral density, $\sigma(x, \omega)$, and integrated in frequency space to get the energy density, $E(t, x)$; then in section 4 we integrated over $x$ to get a total energy. Here we shall perform the integrations in the opposite order. Looking at the spectral and eigenvalue densities is interesting because (unlike most problems) the image method determines them exactly, and the eigenvalues are known, so that one can directly compare the eigenvalue densities. Knowing the eigenvalues, one can then sum over the frequencies, in one's favorite regularization scheme, to get the total energy in the traditional Casimir manner, but we shall not do that explicitly.

The local spectral density (and hence all the other quantities) is divided into three qualitatively different parts in the defining formula (9). The eigenvalue density is thus

$$
\rho(\omega)=\int_{0}^{L} \sigma(\omega, x) d x=\rho_{\mathrm{Weyl}}(\omega)+\rho_{\mathrm{per}}(\omega)+\rho_{\mathrm{bdry}}(\omega),
$$


where

$$
\rho_{\mathrm{Weyl}}(\omega)=\int_{0}^{L} \sigma_{\mathrm{Weyl}} d x=\int_{0}^{L} \frac{\omega}{\pi \kappa} d x=\frac{L \omega}{\pi \kappa},
$$

and similarly

$$
\begin{aligned}
& \rho_{\text {per }}(\omega)=\frac{2 L \omega}{\pi \kappa} \sum_{n=1}^{\infty}(-1)^{n(l+r)} \cos (2 \kappa n L) \\
& \rho_{\text {bdry }}(\omega)=\frac{(-1)^{l}}{2 \pi} \sum_{n=-\infty}^{\infty} \frac{(-1)^{n(l+r)} \omega}{\kappa^{2}}[\sin (2 \kappa L(n+1))-\sin (2 \kappa L n)]
\end{aligned}
$$

where $\kappa=\sqrt{\omega^{2}-m^{2}}$. The eigenvalue counting function equals zero for $\omega<m$ and $\int_{0}^{\omega} \rho(\tilde{\omega}) d \tilde{\omega}$ for $\omega>m$. Therefore, it is (for $\omega>m$ )

$$
\begin{aligned}
N_{\text {Weyl }}(\omega) & =\frac{L}{\pi} \int_{0}^{\kappa} \frac{\omega}{\tilde{\kappa}} \cdot \frac{\tilde{\kappa}}{\omega} d \tilde{\kappa}=\frac{L \kappa}{\pi}=\frac{L \sqrt{\omega^{2}-m^{2}}}{\pi}, \\
N_{\text {per }}(\omega) & =\frac{2 L}{\pi} \sum_{n=1}^{\infty}(-1)^{n(l+r)} \int_{0}^{\kappa} \frac{\sqrt{\tilde{\kappa}^{2}+m^{2}}}{\tilde{\kappa}} \frac{\tilde{\kappa}}{\sqrt{\tilde{\kappa}^{2}+m^{2}}} \cos (2 n L \tilde{\kappa}) d \tilde{\kappa} \\
& =\frac{1}{\pi} \sum_{n=1}^{\infty} \frac{(-1)^{n(l+r)}}{n} \sin (2 n L \kappa),
\end{aligned}
$$

and similarly

$N_{\text {bdry }}(\omega)=\frac{(-1)^{l}}{2 \pi} \sum_{n=-\infty}^{\infty}(-1)^{n(l+r)} \int_{0}^{\tilde{\kappa}} \frac{1}{\tilde{\kappa}}[\sin (2 \tilde{\kappa} L(n+1))-\sin (2 \tilde{\kappa} n L)] d \tilde{\kappa}$.

The Fourier series in (31) can be evaluated as in [5] to a sawtooth function, given in (35)-(36) below. It is easy to see (as at the end of section 4) that $N_{\text {bdry }}=0$ if $l+r$ is odd (that is, equals 1). When $l+r$ is even, we manipulate (32) to the form

$$
N_{\text {bdry }}(\omega)=\frac{(-1)^{l}}{\pi} \lim _{n \rightarrow \infty} \int_{0}^{\kappa} \frac{\sin (2 \kappa L n)}{\kappa} d \kappa
$$

and hence

$$
N_{\text {bdry }}(\omega)=\frac{(-1)^{l}}{\pi} \lim _{n \rightarrow \infty} \int_{0}^{2 n L \kappa} \frac{\sin \tilde{z}}{\tilde{z}} d \tilde{z}=\frac{(-1)^{l}}{\pi} \int_{0}^{\infty} \frac{\sin z}{z} d z=\frac{(-1)^{l}}{2} .
$$

Adding the three counting functions gives

$$
N(\omega)=\frac{L}{\pi} \sqrt{\omega^{2}-m^{2}}+N_{\mathrm{per}}(\omega)+\frac{(-1)^{l}}{2} \delta_{l+r, 1} \quad(\text { for } \omega>m),
$$

where

$$
\begin{aligned}
& N_{\text {per }}(\omega)=\frac{1}{2}-\frac{L \kappa}{\pi} \quad \text { if } l+r \text { is even and } 0<\kappa<\frac{\pi}{L}, \\
& N_{\text {per }}(\omega)=-\frac{L \kappa}{\pi} \quad \text { if } l+r \text { is odd and }-\frac{\pi}{2 L}<\kappa<\frac{\pi}{2 L}
\end{aligned}
$$

both functions are extended periodically to all other intervals on the positive axis of length $\frac{\pi}{L}$ in the variable $\kappa$. 
We now check that $N(\omega)$ is indeed the number of eigenvalues less than or equal to $\omega^{2}$. The true counting function must be 0 for $\omega<m$ and constant and integer-valued on the interval between two eigenvalues. Comparing (34) with (35)-(36), we see that $N$ is indeed constant except at the places where $N_{\text {per }}$ has a discontinuity. At each such

point, $N_{\text {per }}$ jumps from $-\frac{1}{2}$ to $+\frac{1}{2}$, indicating the addition of one new eigenvalue. In the odd case, these points occur at

$$
\kappa=\left(n-\frac{1}{2}\right) \frac{\pi}{L}, \quad \omega^{2}=\left(n-\frac{1}{2}\right)^{2}\left(\frac{\pi}{L}\right)^{2}+m^{2} \quad(n=1,2, \ldots),
$$

and immediately to the right of such a point, $N(\omega)$ evaluates to

$$
\frac{L \kappa}{\pi}+\frac{1}{2}=\left(n-\frac{1}{2}\right)+\frac{1}{2}=n \text {. }
$$

That is, the jumps occur at the correct eigenvalues of the mixed Dirichlet-Neumann problem, and $N$ counts them correctly. In the even case, the jumps are at numbers of the form

$$
\kappa=\frac{n \pi}{L}, \quad \omega^{2}=\left(\frac{n \pi}{L}\right)^{2}+m^{2},
$$

and the limit from the right is

$$
N(\omega)=\frac{L \kappa}{\pi}+\frac{1}{2}+\frac{(-1)^{l}}{2}= \begin{cases}n & \text { if } l=1 \\ n+1 & \text { if } l=0 .\end{cases}
$$

That is, we get the correct eigenvalues for the Dirichlet and Neumann problems, including the extra eigenvalue at $n=0, \omega=m$, in the Neumann case; $N_{\text {per }}$ and $N_{\text {bdry }}$ conspire beautifully to make things come out right at the bottom of the spectrum.

\section{Pressure}

Because of the need to deal with spatial derivatives, the spectral density $\sigma(\omega, x)$ is not convenient for calculating the expectation value of the pressure, $p \equiv\left\langle T_{11}\right\rangle$. Therefore, we revert to the formalism of the cylinder kernel,

$$
\bar{T}\left(t, \mathbf{r}, \mathbf{r}^{\prime}\right)=-\sum_{n=1}^{\infty} \frac{1}{\omega_{n}} \phi_{n}(\mathbf{r}) \phi_{n}\left(\mathbf{r}^{\prime}\right)^{*} e^{-\omega_{n} t}
$$

in terms of the eigenfrequencies and eigenfunctions of the cavity. The cylinder kernel for the massive field in infinite space is [4, (2.2), (3.1)-(3.2)]

$$
\begin{aligned}
\bar{T}_{\infty}(t, x, y) & =-\frac{1}{2 \pi} \int_{-\infty}^{\infty} d \omega \frac{e^{-i \omega t}}{\sqrt{\omega^{2}+m^{2}}} e^{-\sqrt{\omega^{2}+m^{2}}|x-y|} \\
& =-\frac{1}{\pi} K_{0}\left(m \sqrt{t^{2}+(x-y)^{2}}\right) .
\end{aligned}
$$

The kernel for the Casimir slab is then formed by the same construction as in (2), which again generates Weyl, periodic, and boundary terms. Here and henceforth we confine attention to the pure Dirichlet case $(l=r=0)$. 
The fundamental formulas for the energy density and the pressure in terms of the field are (C.3) and (C.4) in Appendix C, from which the formulas for the vacuum expectation values in terms of $\bar{T}$ are

$$
\begin{aligned}
& E(t, x)=-\frac{1}{2} \frac{\partial^{2} \bar{T}}{\partial t^{2}}+\frac{\beta}{2}\left(\frac{\partial^{2} \bar{T}}{\partial x^{2}}+\frac{\partial^{2} \bar{T}}{\partial y^{2}}+2 \frac{\partial^{2} \bar{T}}{\partial x \partial y}\right), \\
& p(t, x)=\frac{1}{8}\left(\frac{\partial^{2} \bar{T}}{\partial x^{2}}+\frac{\partial^{2} \bar{T}}{\partial y^{2}}-2 \frac{\partial^{2} \bar{T}}{\partial x \partial y}\right),
\end{aligned}
$$

where it is understood, formally, that $y$ is set equal to $x$ and $t$ to 0 at the end; $\beta=\xi-\frac{1}{4}$ is the curvature (or conformal) coupling constant, hitherto assumed to be 0 . In arriving at (41)-(42) several routine intermediate steps have been omitted: Passing from the expectation of the product of two fields to $\bar{T}$ requires inserting a compensating factor $\frac{1}{2}$; field products need to be symmetrized; physical time derivatives need to be converted to $t$ derivatives, and in that process $\phi_{0}^{2}$ can be converted to $-\phi \phi_{00}$, so that, in particular, the $\beta$ term in $p$ turns out to vanish identically.

The pressure function for the Weyl term, according to (42) and (40) and the discussion at the end of Appendix B, is given by

$$
\begin{array}{r}
p_{\text {Weyl }}(t, x, y)=\frac{m}{2 \pi}\left(\frac{(t+x-y)(t-x+y) K_{1}\left(m \sqrt{t^{2}+(x-y)^{2}}\right)}{\left(t^{2}+(x-y)^{2}\right)^{3 / 2}}\right. \\
\left.-\frac{m(x-y)^{2} K_{0}\left(m \sqrt{t^{2}+(x-y)^{2}}\right)}{t^{2}+(x-y)^{2}}\right) .
\end{array}
$$

When $y=x$ it simplifies to

$$
p_{\text {Weyl }}(t)=\frac{m}{2 \pi t} K_{1}(m t) \text {. }
$$

The periodic terms are calculated similarly:

$$
p_{\text {per }}(t, x, y)=-\frac{1}{\pi} \frac{d^{2}}{d x^{2}} \sum_{n=1}^{\infty} K_{0}\left(m \sqrt{(x-y+2 n L)^{2}+t^{2}}\right) .
$$

After setting $y=x$ and suppressing the redundant argument, we get

$$
\begin{aligned}
p_{\text {per }}(t, x)=-\frac{m}{\pi} \sum_{n=1}^{\infty}( & \frac{4 L^{2} m n^{2} K_{0}\left(m \sqrt{4 L^{2} n^{2}+t^{2}}\right)}{4 L^{2} n^{2}+t^{2}} \\
& \left.-\frac{(t-2 L n)(2 L n+t) K_{1}\left(m \sqrt{4 L^{2} n^{2}+t^{2}}\right)}{\left(4 L^{2} n^{2}+t^{2}\right)^{3 / 2}}\right)
\end{aligned}
$$

(which actually is independent of $x$ ). In fact, here we can immediately set $t=0$, because there is no divergence in that limit:

$$
\begin{aligned}
p_{\text {per }}(0, x) & =-\frac{m}{\pi} \sum_{n=1}^{\infty}\left(\frac{K_{1}(2 m L n)}{2 L n}+m K_{0}(2 m L n)\right) \\
& =\frac{m^{2}}{\pi} \sum_{n=1}^{\infty} K_{1}^{\prime}(2 m L n)
\end{aligned}
$$


(by (B.3)). The negative of the periodic pressure (47) correctly matches the derivative with respect to $L$ of the total periodic energy, which is $L$ times quantity (16):

$$
\begin{aligned}
-\frac{d}{d L} \bar{E}_{\mathrm{per}}(0) & =\frac{d}{d L}\left(\frac{1}{\pi} \sum_{n=1}^{\infty} m^{2} \frac{K_{1}(2 m L n)}{2 m n}\right) \\
& =\frac{m^{2}}{\pi} \sum_{n=1}^{\infty} K_{1}^{\prime}(2 m L n)=p_{\mathrm{per}}(0,0) \text { or } p_{\mathrm{per}}(0, L) .
\end{aligned}
$$

The argument at the end of Appendix B shows that the boundary terms in the pressure vanish:

$$
p_{\text {bdry }}(t, x, y)=0 .
$$

This result is analogous to the vanishing of $p_{3}$ on p. 5 of [11]; it reflects the fact that moving the boundary does not change the boundary energy.

\section{Conformal correction to the energy}

We digress to discuss the " $\beta$ terms" in (41). The same argument from Appendix B shows that the periodic and Weyl $\beta$ terms add to 0 , the sign change on the third term being compensated by the replacement of $x+y$ by $x-y$, whereas the boundary $\beta$ terms are nonzero, in close analogy with (45)-(46):

$$
\begin{aligned}
\Delta E_{\mathrm{bdry}}^{\beta}(t, x) & =-\frac{2 \beta m}{\pi} \sum_{n=-\infty}^{\infty}\left(\frac{m(2(L n+x))^{2} K_{0}\left(m \sqrt{t^{2}+(2(L n+x))^{2}}\right)}{(2(L n+x))^{2}+t^{2}}\right. \\
& \left.+\frac{(2(\operatorname{Ln}+x)-t)(2(\operatorname{Ln}+x)+t) K_{1}\left(m \sqrt{t^{2}+(2(L n+x))^{2}}\right)}{\left((2(L n+x))^{2}+t^{2}\right)^{3 / 2}}\right) .
\end{aligned}
$$

Combining (50) with (17), we get

$$
\begin{aligned}
E_{\mathrm{bdry}}^{\beta}(0, x)= & E_{\mathrm{bdry}}(0, x)+\Delta E_{\mathrm{bdry}}^{\beta}(0, x) \\
= & -\frac{1}{\pi}\left(\frac{1}{2}+2 \beta\right) \sum_{n=-\infty}^{\infty} \frac{m}{2|x+n L|} K_{1}(2 m|x+n L|) \\
& -\frac{2 \beta m^{2}}{\pi} \sum_{n=-\infty}^{\infty} K_{0}(2 m|x+n L|) .
\end{aligned}
$$

If $\beta=-\frac{1}{4}(\xi=0)$, which counts as both conformal and minimal coupling in space-time dimension 2 , then the first term in (51) vanishes. The surviving term is less singular at the boundary, and it vanishes when $m=0$, as expected for a conformally coupled massless field at a flat (here, 0 -dimensional) boundary. 


\section{Asymptotics of the pressure}

\subsection{Small $t$ or small $m$}

From (44) and (B.1) we have

$$
p_{\text {Weyl }}(t) \sim \frac{1}{2 \pi}\left[\frac{1}{t^{2}}+\frac{m^{2}}{2} \ln \left(\frac{m t}{2}\right)+\frac{m^{2}}{4}(2 \gamma-1)\right],
$$

and thus

$$
p_{\text {Weyl }}(t)=\frac{1}{2 \pi t^{2}} \quad(m=0)
$$

These formulas are to be compared with (15) and (18). In fact, we have

$$
\begin{aligned}
& p_{\text {Weyl }}(t)-E_{\text {Weyl }}(t) \sim \frac{m^{2}}{2 \pi}\left[\ln \left(\frac{m t}{2}\right)+\gamma\right], \\
& p_{\text {Weyl }}(t)+E_{\text {Weyl }}(t) \sim \frac{1}{\pi}\left[\frac{1}{t^{2}}-\frac{m^{2}}{4}\right] .
\end{aligned}
$$

From (54) we see that the zero-point stress tensor of the massless theory, with the $t$ cutoff, is traceless $\left(T_{\mu}^{\mu}=-E+p=0\right)$, as befits a conformally invariant theory. On the other hand, (55) shows that this stress tensor does not satisfy the "principle of virtual work" (energy-pressure balance) [16, 11], $p=-\frac{d \bar{E}}{d L}$ (which is $-E$ in this case). A cutoff procedure that respects Lorentz invariance [17] must yield a zero-point stress proportional to the metric tensor ("dark energy"), replacing (155) by 0 but destroying the tracelessness (unless it makes $p$ and $E$ identically 0 ).

For the periodic term we have already taken the cutoff away at (47), so the only remaining task is to check the massless limit in analogy with section 3.2, In the middle member of (47), the first term is the same as (16) (in the Dirichlet case), and the second term can be shown to vanish as $m \rightarrow 0$ by [14, (2.10)]. Again $p=E$ in the massless limit. But in this case we also have the right pressure balance:

$$
p_{\text {per }}(0)=+E_{\text {per }}(0)=-\frac{\pi}{24 L^{2}}=-\frac{d}{d L}\left(L E_{\text {per }}\right) \quad(m=0) .
$$

We have already seen that the boundary pressure vanishes identically (as does the $L$ derivative of the boundary energy) and that the conformally coupled boundary energy density, (51), vanishes when $m=0$, as does the "renormalized" boundary energy, (24).

\subsection{Supermassive limit}

As $m \rightarrow \infty$, the periodic pressure (47) approaches 0 because each Macdonald function vanishes exponentially rapidly. The same is true of the Weyl pressure (44) so long as $t \neq 0$. The boundary pressure is identically zero. 


\section{Applying the Pauli-Villars method}

In sections 3.1 and 8.1 we have shown that the stress tensor's expectation value has divergences of orders $t^{-2}$ and $\ln t$. In dimension 2 these arise only from the zero-point (Weyl) energy, apart from a caveat about a nonuniform limit at the endpoints in the boundary energy, to which we shall return. The structure is most clearly shown in (54)-(55).

In these sections, the display of formulas with coincident spatial coordinates and small, imaginary time separation is purely for calculational and expository convenience; in principle, the coordinates are arbitrary. It may appear that we have done a kind of "point-splitting" regularization at an intermediate step; this perception is wrong. The spirit of Pauli-Villars regularization is to do the subtractions "at the theory level". In practice, this means that the subtractions involve Green functions as a whole, regarded as distributions (or, in other words, they involve the operators that the Green functions represent). Thus the potential divergences are removed before the issue of evaluating the Green functions at coincident arguments ever arises.

Following Appendix A, consider the effect of superposing the stress tensors for several (or many) values of $m$ :

$$
E=\int E[m] f(m) d m, \quad p=\int p[m] f(m) d m,
$$

where the function or distribution $f$ is independent of $t$. If (A.7) and (A.8) are satisfied, the terms in (55) are obliterated; thus the Weyl part of the vacuum stress satisfies

$$
p_{\text {Weyl }}=-E_{\text {Weyl }}
$$

(a nontrivial result, in view of [17] and [11]). We have already observed (section 8.1) that the periodic and boundary parts of the stress are also nonanomalous, though the relations expressing this health are different from (58) because the respective total energies have different dependences on $L$.

If, in addition,

$$
\int f(m) m^{2} \ln m d m=0,
$$

then (54) is also obliterated. If one requires merely that this logarithmic integral be finite, as in (A.10), then the stress tensor is finite for all $t$ but its Weyl part may be a nontrivial multiple of the metric tensor, a two-dimensional version of the cosmological constant.

The model as it stands is unlikely to be physically realistic, because it contains the effects of unphysical fields with negative energies. Therefore, one studies the effect of taking the unphysical auxiliary masses very large, in hopes that their effects will become unobservable. We verified in sections 3.3 and 8.2 that the periodic and boundary terms vanish in this limit; only the vacuum stresses of the original physical field will survive. If one can guarantee that the integral on the left of (59) remains finite in the limit, then the final theory has no divergences but does have a "cosmological" term with an 
undetermined coefficient. If the logarithmic integral is allowed to grow without bound, to get a finite theory an explicit bare cosmological counterterm must be assumed, but the construction is Lorentz-covariant, unlike (55), the result of an ultraviolet cutoff.

The story is different, however, if we look at the total energy. It is tempting to appeal to (25), but to take the $m$ limit before the $t$ limit would be inconsistent with our treatment of the Weyl term. So, we are stuck with (24), a boundary energy linear in $m$. Recall that it arose because of the nonuniform limiting behaviors of the boundary stress at the endpoints of the interval; in some sense it is concentrated on the endpoints and has become independent of the stress in the interior, which we have succeeded in regularizing. Obliterating it appears to require yet another constraint on the mass distribution $f$.

In conclusion, we have shown that the Pauli-Villars construction is mathematically feasible and eliminates the only pressure anomaly that arises in dimension 2, the direction dependence of Christensen [17]. Physically, whether this maneuver is any more convincing than the "analytic" methods (zeta functions and dimensional regularization) is open to debate. Further philosophical discussion probably should wait until an implementation in four-dimensional space-time, where the anomaly of Estrada et al. [11] will be encountered in the ultraviolet-cutoff theory.

\section{Acknowledgements}

This research was supported by National Science Foundation Grant PHY-0968269, and by the renewed hospitality of the Mathematics Department of Texas A\&M University toward F.D.M. while some of the work was done. We thank Klaus Kirsten and Kim Milton for critical remarks on a preliminary presentation of the results. Mathematica was useful at various stages of the work.

\section{Appendix A. Varieties of Pauli-Villars regularization}

Pauli-Villars regularization introduces auxiliary fields whose divergences have on balance opposite sign from those of the original, physical fields, so that the total predictions of the theory are finite. Usually the auxiliary masses are taken very large, so that the new fields have no noticeable effects on the finite predictions.

The original paper of Pauli and Villars [6] (which remarks, "This method has already a long history,") deals with quantum electrodynamics in Minkowski space-time. Later the method was applied in cosmological contexts [7, 8, 9] and in quantum gravity [10]. There are major differences in philosophy and procedure among these works.

Pauli and Villars distinguish between "realistic" and "formalistic" regularizations. In realistic theories the auxiliary masses are assumed to belong to real (physical) fields, whose vacuum energies for some reason do not all have the same sign; these masses are kept finite. In formalistic theories the auxiliary fields are fictitious, and their masses

are sent to infinity at the end of the calculations. The realistic approach replaces the 
original theory by a new theory; not surprisingly, the results are not unique. In the formalistic approach after the limit of infinite mass, the only surviving ambiguities are those that arise in all renormalization schemes and are proportional to the erstwhile divergences. This is our understanding, in the present context, of the Pauli-Villars ambiguities recently pointed out in [18]. These phenomena are visible below in our two treatments of the Zel'dovich regularization of integrals.

Zel'dovich [7] (whose method is followed by Streeruwitz [8] without much further discussion of its rationale) starts from divergent integrals such as

$$
\lim _{\Lambda \rightarrow \infty} 4 \pi \int_{0}^{\Lambda} \sqrt{p^{2}+m^{2}} p^{2} d p
$$

and postulates a mass distribution function $f(m)$ (possibly a finite sum of delta functions) such that

$$
\int f(m) d m=\int f(m) m^{2} d m=\int f(m) m^{4} d m=0,
$$

so that

$$
\int d m f(m) \int_{0}^{\Lambda} p^{2} d p \sqrt{p^{2}+m^{2}}
$$

has an asymptotic expansion containing no positive powers of $\Lambda$. It may contain terms proportional to

$$
\int f(m) m^{2 p} \ln (\Lambda m) d m \quad(p=0,1,2),
$$

but these are actually independent of $\Lambda$ by virtue of (A.2). Thus, given a fixed $f$ for which the integrals in (A.4) with $\Lambda=1$ converge, the ultraviolet divergences have been eliminated. Because it is not required that the integrals (A.4) equal 0, arbitrary renormalization constants appear.

The intention of Zel'dovich was that $f(m)$ represent a spectrum of real particles, with negative values of $f$ arising from fermions. This theory, therefore, is of the "realistic" type; it is a forerunner of supersymmetry. Zel'dovich's main motivation was to produce a nonzero, but finite, cosmological constant from the integral

$$
\int f(m) m^{4} \ln m d m
$$

Note, however, that there is a possibility of creating a "formalistic" theory by moving the support of the negative part (at least) of $f$ off to infinity at the end of the argument, provided that any integrals like (A.5) that arise still converge in this limit. It is not immediately obvious that this can be done, and especially whether the finite limiting values of the renormalization constants can be different from 0 . It is rather easy to see that the minimal finite sum consistent with (A.2) will not work: Take

$$
f(m)=\delta(m)-\delta\left(m-m_{1}\right)+\delta\left(m-m_{2}\right)-\delta\left(m-m_{3}\right),
$$


where the first term represents the physical field (taken here to be massless for simplicity) and the other three masses are to be taken to $\infty$. Consider for simplicity a twodimensional space-time, so that the only constraints from (A.2) and (A.4) that must be satisfied are

$$
\begin{aligned}
& \int f(m) d m=0, \\
& \int f(m) m^{2} d m=0, \\
& \int f(m) \ln m d m<\infty, \\
& \int f(m) m^{2} \ln m d m<\infty .
\end{aligned}
$$

(In (A.9) the term with $m=0$ should be omitted. The precise meaning of (A.9) and (A.10) is that the sums remain bounded as the $m_{j}$ go to infinity.) It is clear that to satisfy (A.7) the total number of masses must be even, and then to satisfy (A.8) also, the number must be at least 4 . Let us first consider the case $m_{1}=m_{3}$; then by (A.6), (A.8) becomes

$$
m_{2}^{2}=2 m_{1}^{2} .
$$

Then (A.10) states that

$$
m_{2}^{2} \ln m_{2}-2 m_{1}^{2} \ln m_{1}=2 m_{1}^{2} \ln \sqrt{2}
$$

is bounded as $m_{1} \rightarrow \infty$, which is false. Now try $m_{3}=\nu m_{1}$, with $\nu>1$ : After some algebra one gets from (A.8) and (A.10) the same sort of contradiction, unless

$$
F(\nu) \equiv\left(1+\nu^{2}\right) \ln \left(1+\nu^{2}\right)-2 \nu^{2} \ln \nu=0 .
$$

But $F(1)=2 \ln 2>0$ and

$$
F^{\prime}(\nu)=2 \nu \ln \left(1+\nu^{2}\right)-2 \nu \ln \nu^{2}>0,
$$

so splitting the masses can only make the problem worse.

Bernard and Duncan [9] take a formalistic approach from the beginning. They consider a two-dimensional cosmological space-time. Unlike [7, 8], who impose (A.3) a posteriori, they start with a Lagrangian and explicitly construct a Fock space. Their negative-energy fields (corresponding to masses $m_{1}$ and $m_{3}$ in the foregoing) are not ordinary fermion fields, but anticommuting scalar fields producing states with negative metric. In the infinite-mass limit this sector of the state space decouples, leaving a unitary dynamics in a Hilbert space. This construction apparently requires $m_{1}=m_{3}$, so the mass spectrum in [9] is the same as (A.6), except that they allow the physical field to have a mass, $\mu$; then $m_{2}^{2}=2 M^{2}-\mu^{2}$ and (in effect) $m_{1}^{2}=m_{3}^{2}=M^{2}$. Thus (A.7) and (A.8) are satisfied. It turns out that (A.9) is unnecessary because of the triviality of two-dimensional gravity. But (A.10) is not satisfied in the limit; instead, Bernard and Duncan explicitly introduce a cosmological counterterm to cancel this divergence. They remark that the analogous construction in dimension 4 would require 
seven regulator fields (counting the complex anticommuting ones twice) and (as usual in four-dimensional gravity) four counterterms. (Without the constraint that both masses associated with an anticommuting field be the same, the three regulator masses in (A.6) would be enough to satisfy (A.2).)

In the Bernard-Duncan approach, then, the Pauli-Villars construction does not, by itself, remove infinities, but it does achieve covariance: Divergences in the limit of large cutoff, $\Lambda$, are replaced by divergences in the limit of large $M$. The regulated (finite- $M$ ) expressions are free of the direction dependence [17] and resulting pressure anomalies [11] characteristic of point-splitting calculations of the stress tensor.

Anselmi [10] takes the further step of cancelling the large- $M$ divergences by adding still more regulator fields. He requires that the logarithmic sums (A.4) vanish. He inserts the regulator fields into a path integral in a nonstandard way, which permits (in effect) spectra like (A.6) with coefficients not necessarily equal to \pm 1 . This additional freedom allows the conditions to be satisfied by solving a linear system for those coefficients, instead of the nonlinear system for the masses; this significantly simplifies the study of the existence question. The result is that, with enough regulator fields, a formalistic formulation without counterterms is achieved. (Nevertheless, because of the need to modify (A.4) for $p=0$ when the physical field is massless, the logarithmic divergences inevitably result in two arbitrary renormalized coupling constants in the final equation of motion of the gravitational field. In the present paper this complication does not concern us.)

\section{Appendix B. Calculus with Macdonald functions}

In [13] or any similar reference one finds the approximations

$$
K_{1}(z)=\frac{1}{z}+\frac{z}{2} \ln \frac{z}{2}+\left(\gamma-\frac{1}{2}\right) \frac{z}{2}+O\left(z^{3} \ln z\right)
$$

for small $z$ and

$$
K_{\nu}(z) \sim \sqrt{\frac{\pi}{2 z}} e^{-z}
$$

for large $z$.

Derivatives can be eliminated by [13, (8.486.12)]

$$
K_{0}^{\prime}(z)=-K_{1}(z), \quad K_{1}^{\prime}(z)=-\frac{1}{z} K_{1}(z)-K_{0}(z) .
$$

The integral

$$
\int_{t}^{\infty} \frac{K_{1}(m u)}{\sqrt{u^{2}-t^{2}}} d u=\frac{\pi e^{-m t}}{2 m t}
$$

does not appear in [13] but is known to Mathematica and can be deduced from [13, (6.596.3)].

In section 6 and section 7 we repeatedly encounter second derivatives of

$$
K_{0}\left(m \sqrt{(x \pm y+2 n L)^{2}+t^{2}}\right) .
$$


The results are simplified by (B.3) and further simplified by cancellations and combinations: Looking, for example, at (42), one can show that the first two terms are equal and their sum is equal to the third term up to sign. Thus the whole expression vanishes if the variable sign in (B.5) is + and equals 4 times the first term if that sign is - . For the $\beta$ terms in (41) the role of the sign is precisely the reverse.

\section{Appendix C. The stress tensor in dimension 2}

The general form of the scalar stress tensor, defined by variation of the gravitational Lagrangian with respect to the metric, is given (in the sign convention where $g_{00}<0$ ) in [19, 17, 20]. After specializing to flat space (and glossing over operator symmetrizations), it is

$$
\begin{aligned}
T_{\mu \nu} & =(1-2 \xi) \phi_{\mu} \phi_{\nu}+\left(2 \xi-\frac{1}{2}\right) g_{\mu \nu} \phi_{\sigma} \phi^{\sigma}-2 \xi \phi \phi_{\mu \nu}+2 \xi g_{\mu \nu} \phi \phi_{\sigma}^{\sigma}-\frac{1}{2} m^{2} g_{\mu \nu} \phi^{2} \\
& =\frac{1}{2}\left[\phi_{\mu} \phi_{\nu}-\phi \phi_{\mu \nu}+g_{\mu \nu} \phi \phi_{\sigma}^{\sigma}-m^{2} g_{\mu \nu} \phi^{2}\right]+2 \beta\left[-\phi_{\mu} \phi_{\nu}+g_{\mu \nu} \phi_{\sigma} \phi^{\sigma}-\phi \phi_{\mu \nu}+g_{\mu \nu} \phi \phi_{\sigma}^{\sigma}\right],
\end{aligned}
$$

where $\xi \equiv \beta+\frac{1}{4}$ is the curvature coupling constant (and indices on $\phi$ denote derivatives). Using the equation of motion, $\phi_{\sigma}^{\sigma}=m^{2} \phi$, to rewrite the first term (but not the second), one arrives at

$$
T_{\mu \nu}=\frac{1}{2}\left[\phi_{\mu} \phi_{\nu}-\phi \phi_{\mu \nu}\right]+2 \beta\left[-\phi_{\mu} \phi_{\nu}+g_{\mu \nu} \phi_{\sigma} \phi^{\sigma}-\phi \phi_{\mu \nu}+g_{\mu \nu} \phi \phi_{\sigma}^{\sigma}\right] .
$$

The advantages of this form are (a) the mass (more generally, a scalar potential [21]) does not appear at all, (b) the first term of $T_{\mu \mu}$ contains only $\mu$ derivatives, and (c) the $\beta$ term is manifestly a total derivative. Specializing henceforth to dimension $1+1$, we have

$$
\begin{aligned}
& T_{00}=\frac{1}{2}\left[\phi_{0}^{2}-\phi \phi_{00}\right]-2 \beta\left[\phi_{1}^{2}+\phi \phi_{11}\right], \\
& T_{11}=\frac{1}{2}\left[\phi_{1}^{2}-\phi \phi_{11}\right]-2 \beta\left[\phi_{0}^{2}+\phi \phi_{00}\right] .
\end{aligned}
$$

\section{References}

[1] Milton K A 2001 The Casimir Effect: Physical Manifestations of Zero-Point Energy Singapore:World Scientific.

[2] Bordag M, Klimchitskaya G L, Mohideen U and Mostepanenko V M 2009 Advances in the Casimir Effect Oxford:Clarendon Press.

[3] Dalvit D, Milonni P, Roberts D and da Rosa F (eds.) 2011 Casimir Physics (Lecture Notes in Physics 834) Berlin:Springer.

[4] Hays P 1979 Vacuum fluctuations of a confined massive field in two dimensions Ann. Phys. 121 $32-46$

[5] Fulling S A 2007 Vacuum energy as spectral geometry SIGMA 3094 arXiv:0706.2831.

[6] Pauli W and Villars F 1949 On the invariant regularization in relativistic quantum theory Rev. Mod. Phys. 21 434-444

[7] Zel'dovich Ya B 1968 The cosmological constant and the theory of elementary particles Sov. Phys. Usp. 11 381-393 [Usp. Fiz. Nauk 95 209-230]

[8] Streeruwitz E 1975 Vacuum fluctuations of a quantized scalar field in a Robertson-Walker universe Phys. Rev. D 11 3378-3383 
[9] Bernard C and Duncan A 1977 Regularization and renormalization of quantum field theory in curved space-time Ann. Phys. 107 201-221

[10] Anselmi D 1993 Covariant Pauli-Villars regularization of quantum gravity at the one-loop order Phys. Rev. D 48 5751-5763

[11] Estrada R, Fulling S A, and Mera F D 2012 Surface vacuum energy in cutoff models: Pressure anomaly and distributional gravitational limit J. Phys. A 45455402

[12] Bender C M and Hays P 1976 Zero-point energy of fields in a finite volume Phys. Rev. D 14 $2622-2632$

[13] Gradshteyn I S and Ryzhik I M 1965 Table of Integrals, Series and Products New York:Academic Press

[14] Kirsten K 1992 Connections between Kelvin functions and zeta functions with applications $J$. Phys. A 25 6297-6305

[15] Titchmarsh E. C. 1939 The Theory of Functions (2nd ed) Oxford:Oxford University Press

[16] Barton G 2004 Casimir's spheres near the Coulomb limit: energy density, pressures and radiative effects J. Phys. A 37 3725-3741

[17] Christensen S M 1976 Vacuum expectation value of the stress tensor in an arbitrary curved background: The covariant point-separation method Phys. Rev. D 14 2490-2501

[18] Kleiss R H P and Janssen T 2014 Ambiguities in Pauli-Villars regularization arXiv:1405.1536.

[19] DeWitt B S 1975 Quantum field theory in curved spacetime Phys. Rep. 19 295-357

[20] Birrell N D and Davies P C W 1982 Quantum Fields in Curved Space Cambridge:Cambridge University Press.

[21] Milton K A, Shajesh K V, Fulling S A, and Parashar P 2014 How does Casimir energy fall? IV Phys. Rev. D 89064027 